Internet Research Task Force (IRTF)

Request for Comments: 7046

Category: Experimental

ISSN : 2070-1721
M. Waehlisch

link-lab \& FU Berlin

T. Schmidt

HAW Hamburg

S. Venaas

Cisco Systems

December 2013

\title{
A Common API for Transparent Hybrid Multicast
}

\section{Abstract}

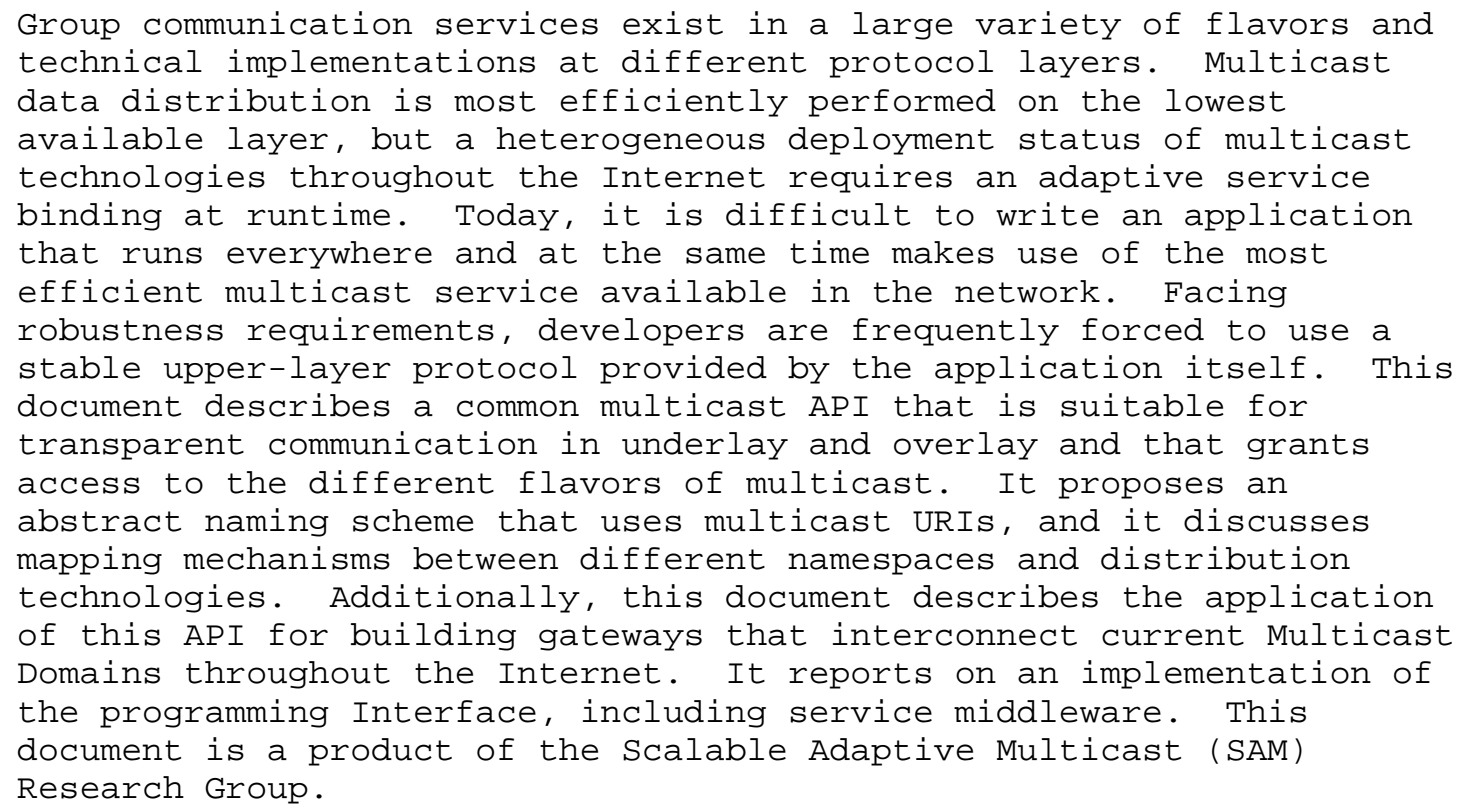




\section{Status of This Memo}

This document is not an Internet Standards Track specification; it is published for examination, experimental implementation, and evaluation.

This document defines an Experimental Protocol for the Internet community. This document is a product of the Internet Research Task Force (IRTF). The IRTF publishes the results of Internet-related research and development activities. These results might not be suitable for deployment. This RFC represents the consensus of the Scalable Adaptive Multicast Research Group of the Internet Research Task Force (IRTF). Documents approved for publication by the IRSG are not a candidate for any level of Internet Standard; see Section 2 of RFC 5741 .

Information about the current status of this document, any errata, and how to provide feedback on it may be obtained at http://www.rfc-editor.org/info/rfc7046.

Copyright Notice

Copyright (c) 2013 IETF Trust and the persons identified as the document authors. All rights reserved.

This document is subject to BCP 78 and the IETF Trust's Legal Provisions Relating to IETF Documents

(http://trustee.ietf.org/license-info) in effect on the date of publication of this document. Please review these documents carefully, as they describe your rights and restrictions with respect to this document.

Table of Contents

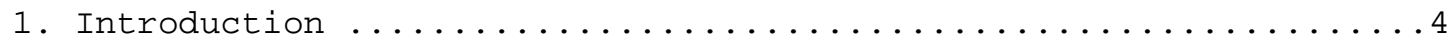

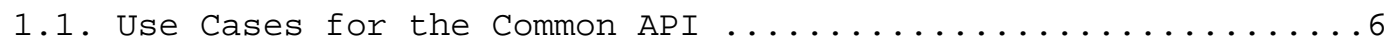

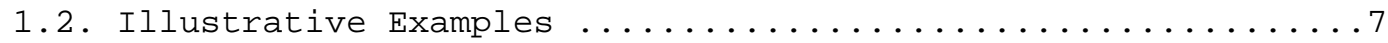
1.2.1. Support of Multiple Underlying Technologies ......7

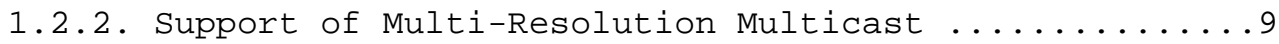

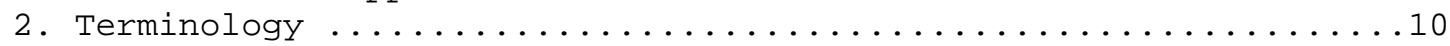

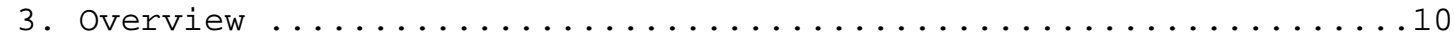

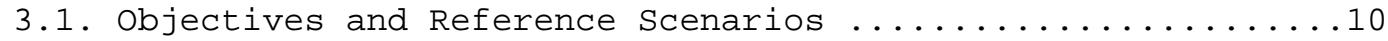

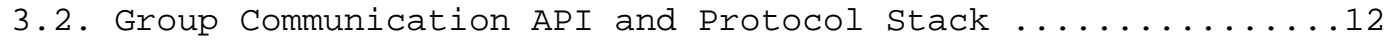

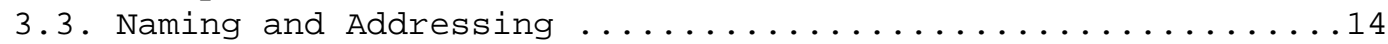

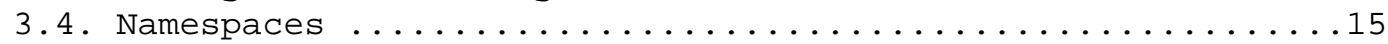




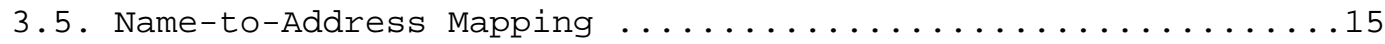

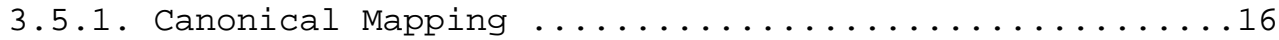

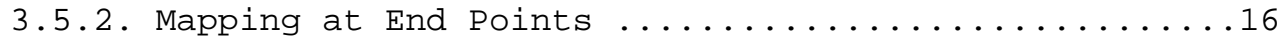

3.5.3. Mapping at Inter-Domain Multicast Gateways ......16

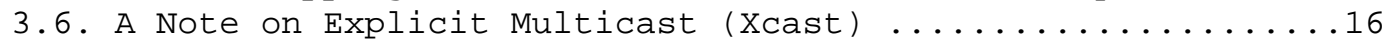

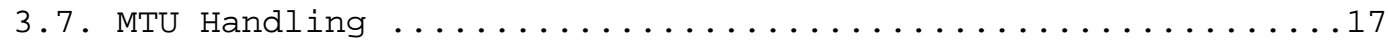

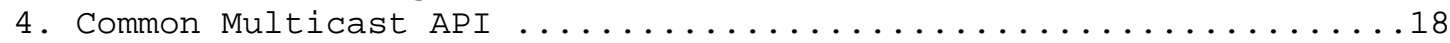

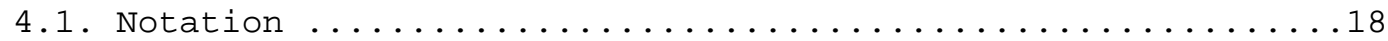

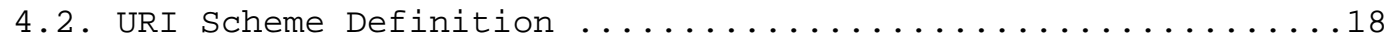

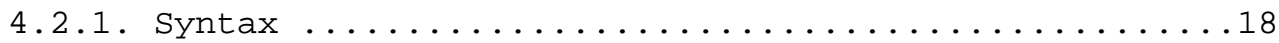

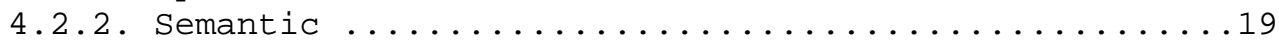

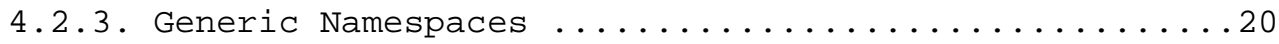

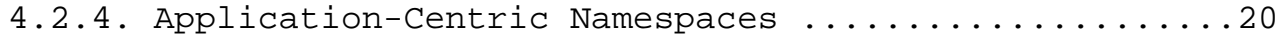

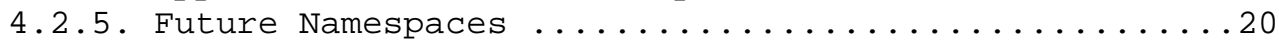

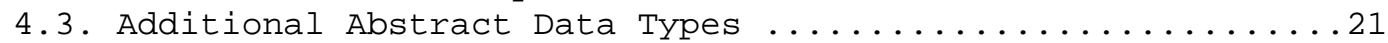

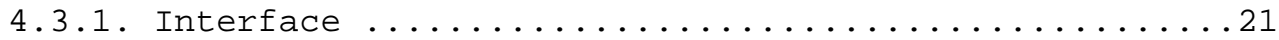

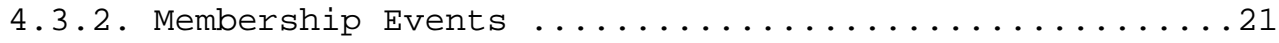

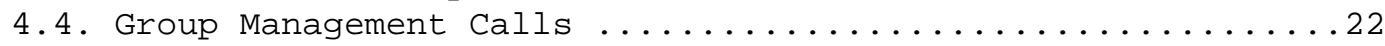

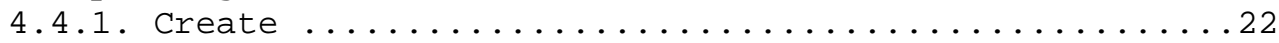

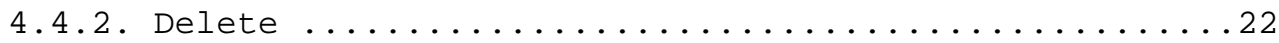

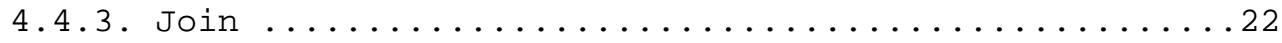

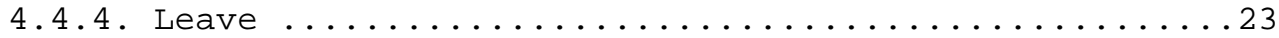

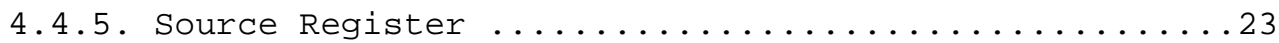

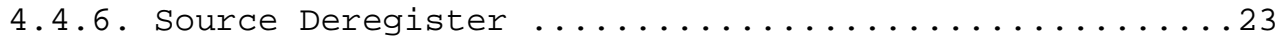

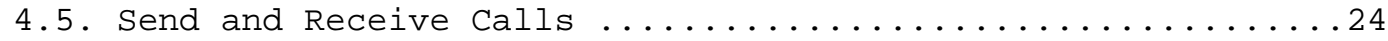

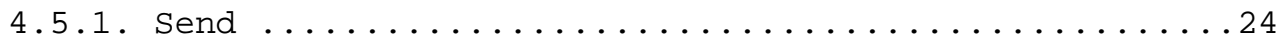

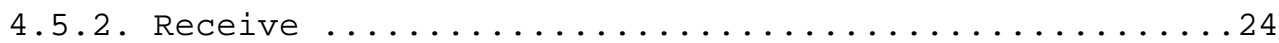

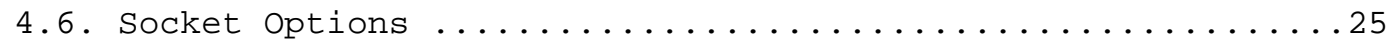

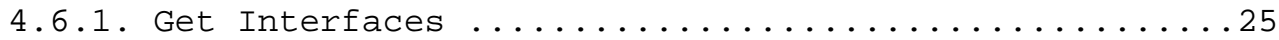

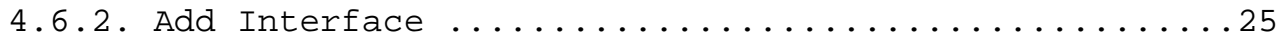

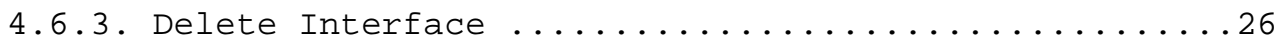

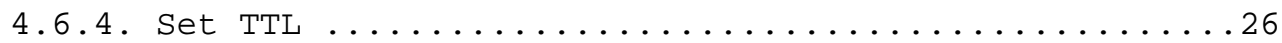

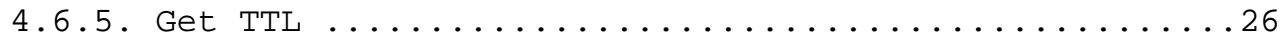

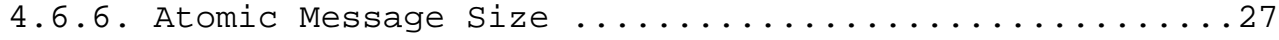

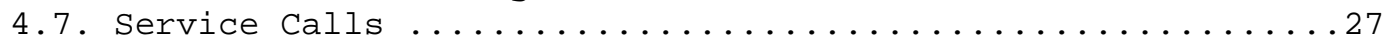

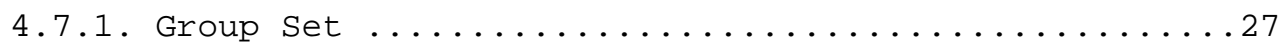

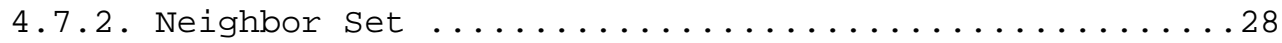

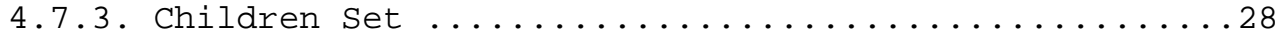

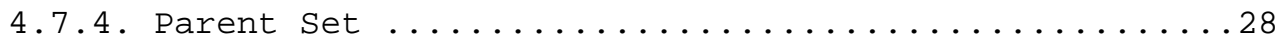

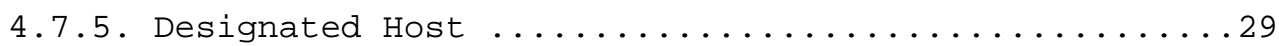

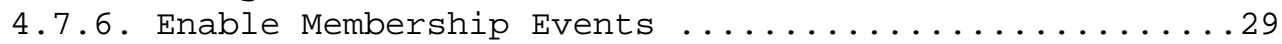

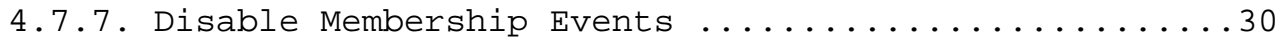

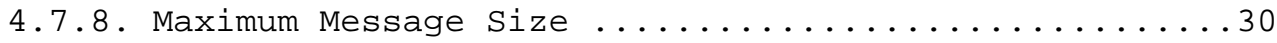

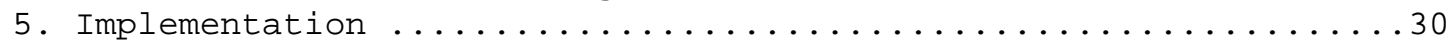

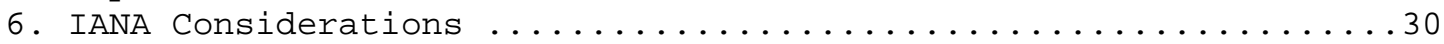

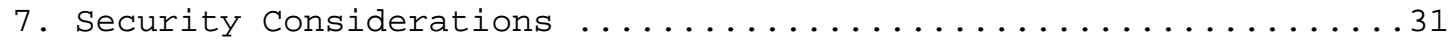

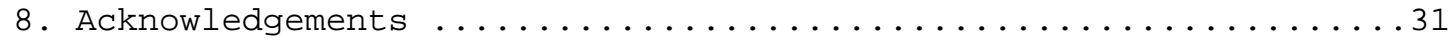




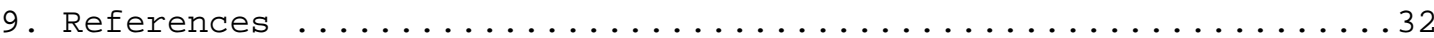

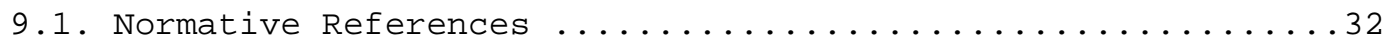

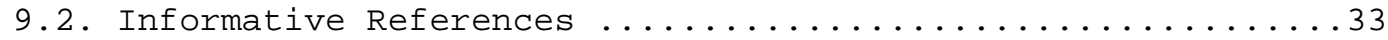

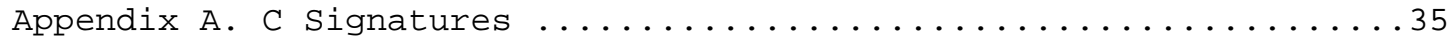

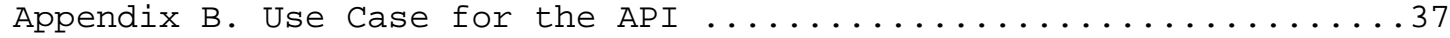

Appendix C. Deployment Use Cases for Hybrid Multicast ............38

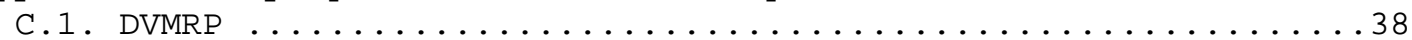

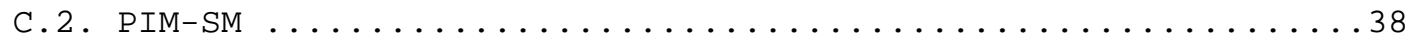

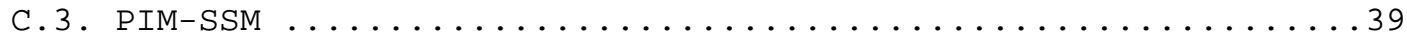

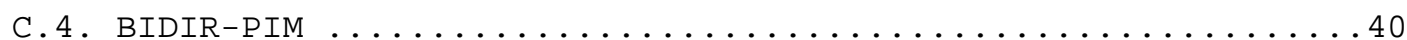

1. Introduction

Currently, group application programmers need to choose the distribution technology that the application will require at runtime. There is no common communication Interface that abstracts multicast transmission and subscriptions from the deployment state at runtime, nor has the use of DNS for Group Addresses been established. The standard multicast socket options [RFC3493] [RFC3678] are bound to an IP version by not distinguishing between the naming and addressing of multicast identifiers. Group communication, however,

- is commonly implemented in different flavors, such as any-source multicast (ASM) vs. source-specific multicast (SSM),

o is commonly implemented on different layers (e.g., IP vs. application-layer multicast), and

- may be based on different technologies on the same tier, as seen with IPv4 vs. IPv6.

The objective of this document is to provide for programmers a universal access to group services.

Multicast application development should be decoupled from technological deployment throughout the infrastructure. It requires a common multicast API that offers calls to transmit and receive multicast data independent of the supporting layer and the underlying technological details. For inter-technology transmissions, a consistent view of multicast states is needed as well. This document describes an abstract group communication API and core functions necessary for transparent operations. Specific implementation guidelines with respect to operating systems or programming languages are out of scope for this document. 
In contrast to the standard multicast socket Interface, the API introduced in this document abstracts naming from addressing. Using a multicast address in the current socket API predefines the corresponding routing layer. In this specification, the multicast name used for joining a group denotes an application-layer data stream that is identified by a multicast URI, independent of its binding to a specific distribution technology. Such a Group Name can be mapped to variable routing identifiers.

The aim of this common API is twofold:

- Enable any application programmer to implement group-oriented data communication independent of the underlying delivery mechanisms. In particular, allow for a late binding of group applications to multicast technologies that makes applications efficient but robust with respect to deployment aspects.

- Allow for flexible namespace support in group addressing and thereby separate naming and addressing (or routing) schemes from the application design. This abstraction not only decouples programs from specific aspects of underlying protocols but may open application design to extend to specifically flavored group services.

Multicast technologies may be of various peer-to-peer kinds, IPv4 or IPv6 network-layer multicast, or implemented by some other application service. Corresponding namespaces may be IP addresses or DNS naming, overlay hashes, or other application-layer group identifiers like <sip: @peanuts.org>, but they can also be names independently defined by the applications. Common namespaces are introduced later in this document but follow an open concept suitable for further extensions.

This document also discusses mapping mechanisms between different namespaces and forwarding technologies and proposes expressions of defaults for an intended binding. Additionally, the multicast API provides internal Interfaces to access current multicast states at the host. Multiple multicast protocols may run in parallel on a single host. These protocols may interact to provide a gateway function that bridges data between different domains. The usage of this API at gateways operating between current multicast instances throughout the Internet is described as well. Finally, a report on an implementation of the programming Interface, including service middleware, is presented. 
This document represents the consensus of the SAM Research Group. It has been reviewed by the Research Group members active in the specific area of work. In addition, this document has been comprehensively reviewed by people who are not "in" the Research Group but are experts in the area.

\subsection{Use Cases for the Common API}

The following generic use cases can be identified; these use cases require an abstract common API for multicast services:

Application Programming Independent of Technologies: Application programmers are provided with group primitives that remain independent of multicast technologies and their deployment in target domains. Thus, for a given application, they can develop a program that will run in every deployment scenario. The use of Group Names in the form of abstract metadata types allows applications to remain namespace-agnostic in the sense that the resolution of namespaces and name-to-address mappings may be delegated to a system service at runtime. Complexity is thereby minimized, as developers need not care about how data is distributed in groups, while the system service can take advantage of extended information of the network environment as acquired at startup.

Global Identification of Groups: Groups can be identified independent of technological instantiations and beyond deployment domains. Taking advantage of the abstract naming, an application can thus match data received from different Interface technologies (e.g., IPv4, IPv6, and overlays) to belong to the same group. This not only increases flexibility -- an application may, for instance, combine heterogeneous multipath streams -- but also simplifies the design and implementation of gateways.

Uniform Access to Multicast Flavors: The URI naming scheme uniformly supports different flavors of group communication, such as any-source multicast and source-specific multicast, and selective broadcast, independent of their service instantiation. The traditional SSM model, for instance, can experience manifold support by directly mapping the multicast URI (i.e., "groupdinstantiation") to an $(S, G)$ state on the IP layer, by first resolving $S$ for a subsequent Group Address query, by transferring this process to any of the various source-specific overlay schemes, or by delegating to a plain replication server. The application programmer can invoke any of these underlying mechanisms with the same line of code. 
Simplified Service Deployment through Generic Gateways: The common multicast API allows for an implementation of abstract gateway functions with mappings to specific technologies residing at the system level. Generic gateways may provide a simple bridging service and facilitate an inter-domain deployment of multicast.

Mobility-Agnostic Group Communication: Group naming and management as foreseen in the common multicast API remain independent of locators. Naturally, applications stay unaware of any mobilityrelated address changes. Handover-initiated re-addressing is delegated to the mapping services at the system level and may be designed to smoothly interact with mobility management solutions provided at the network or transport layer (see [RFC5757] for mobility-related aspects).

\subsection{Illustrative Examples}

\subsubsection{Support of Multiple Underlying Technologies}

On a very high level, the common multicast API provides the application programmer with one single Interface to manage multicast content independent of the technology underneath. Considering the following simple example in Figure 1, a multicast source $S$ is connected via IPv4 and IPv6. It distributes one flow of multicast content (e.g., a movie). Receivers are connected via IPv4/v6 and Overlay Multicast (OM), respectively.

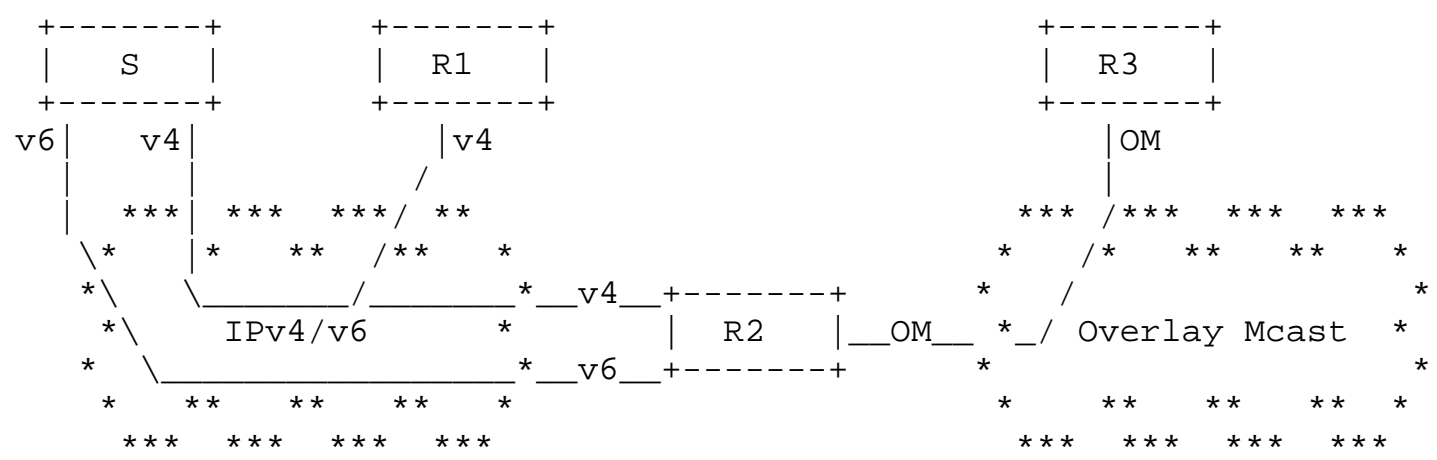

Figure 1: Common Scenario: Source S Sends the Same Multicast Content via Different Technologies

Using the current BSD socket API, the application programmer needs to decide on the IP technologies at coding time. Additional distribution techniques, such as overlay multicast, must be individually integrated into the application. For each technology, the application programmer needs to create a separate socket and 


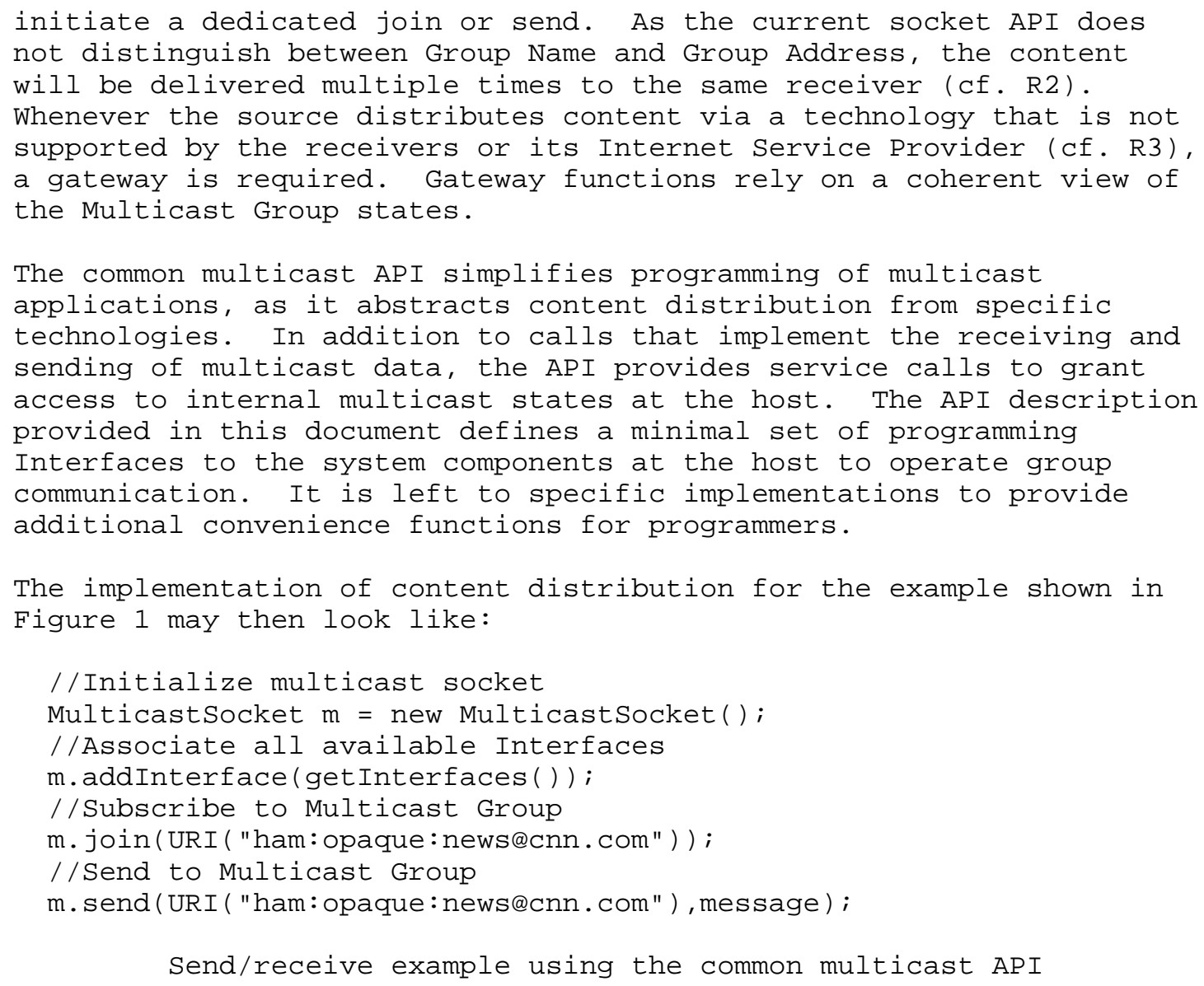


The gateway function for R2 can be implemented by service calls that look like:

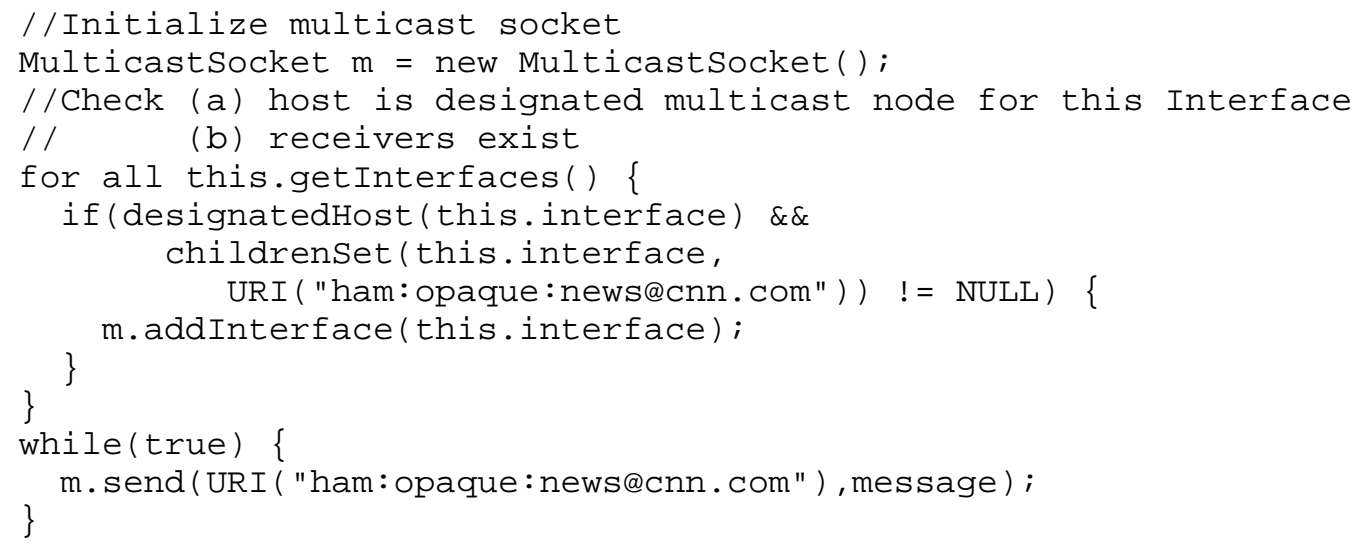

Gateway example using the common multicast API

\subsubsection{Support of Multi-Resolution Multicast}

Multi-resolution multicast adjusts the multicast stream to consider heterogeneous end devices. The multicast data (e.g., available by different compression levels) is typically announced using multiple multicast addresses that are unrelated to each other. Using the common API, multi-resolution multicast can be implemented transparently by an operator with the help of name-to-address mapping, or by systematic naming from a subscriber-centric perspective.

Operator-Centric: An operator deploys a domain-specific mapping. In this case, any multicast receiver (e.g., mobile or DSL user) subscribes to the same multicast name, which will be resolved locally to different multicast addresses. In this case, each Group Address represents a different level of data quality.

Subscriber-Centric: In a subscriber-centric example, the multicast receiver chooses the quality in advance, based on a predefined naming syntax. Consider a layered video stream "blockbuster" available at different qualities Q_i, each of which consists of the base layer plus the sum of EL_j, $j<=i$ enhancement layers. Each individual layer may then be accessible by a name "EL_j.Q_i.blockbuster", $j<=i$, while a specific quality aggregates the corresponding layers to "Q_i.blockbuster", and the full-size movie may be just called "blockbuster". 


\section{Terminology}

This document uses the terminology as defined for the multicast protocols discussed in [RFC2710], [RFC3376], [RFC3810], [RFC4601], and [RFC4604]. In addition, the following terms will be used:

Group Address: A Group Address is a routing identifier. It represents a technological specifier and thus reflects the distribution technology in use. Multicast packet forwarding is based on this address.

Group Name: A Group Name is an application identifier used by applications to manage communication in a Multicast Group (e.g., join/leave and send/receive). The Group Name does not predefine any distribution technologies. Even if it syntactically corresponds to an address, it solely represents a logical identifier.

Multicast Namespace: A Multicast Namespace is a collection of designators (i.e., names or addresses) for groups that share a common syntax. Typical instances of namespaces are IPv4 or IPv6 multicast addresses, overlay group IDs, Group Names defined on the application layer (e.g., SIP or email), or some human-readable string.

Interface: An Interface is a forwarding instance of a distribution technology on a given node, for example, the IP Interface 192.168.1.1 at an IPv4 host, or an overlay routing Interface.

Multicast Domain: A Multicast Domain hosts nodes and routers of a common, single multicast forwarding technology and is bound to a single namespace.

Inter-domain Multicast Gateway (IMG): An IMG is an entity that interconnects different Multicast Domains. Its objective is to forward data between these domains, e.g., between an IP layer and overlay multicast.

\section{Overview}

\subsection{Objectives and Reference Scenarios}

The default use case addressed in this document targets applications that participate in a group by using some common identifier taken from some common namespace. This Group Name is typically learned at runtime from user interaction, such as the selection of an IPTV channel, or from dynamic session negotiations as used with the Session Initiation Protocol (SIP) [RFC3261] or Peer-to-Peer SIP 
(P2PSIP) [SIP-RELOAD], but may as well have been predefined for an application as a common Group Name. Technology-specific system functions then transparently map the Group Name to Group Addresses such that

- programmers can process Group Names in their programs without the need to consider technological mappings that relate to designated deployments in target domains;

- applications can identify packets that belong to a logically named group, independent of the Interface technology used for sending and receiving packets; this shall also hold true for multicast gateways.

This document considers two reference scenarios that cover the following hybrid deployment cases displayed in Figure 2:

1. Multicast Domains running the same multicast technology but remaining isolated, possibly only connected by network-layer unicast.

2. Multicast Domains running different multicast technologies but hosting nodes that are members of the same Multicast Group. 


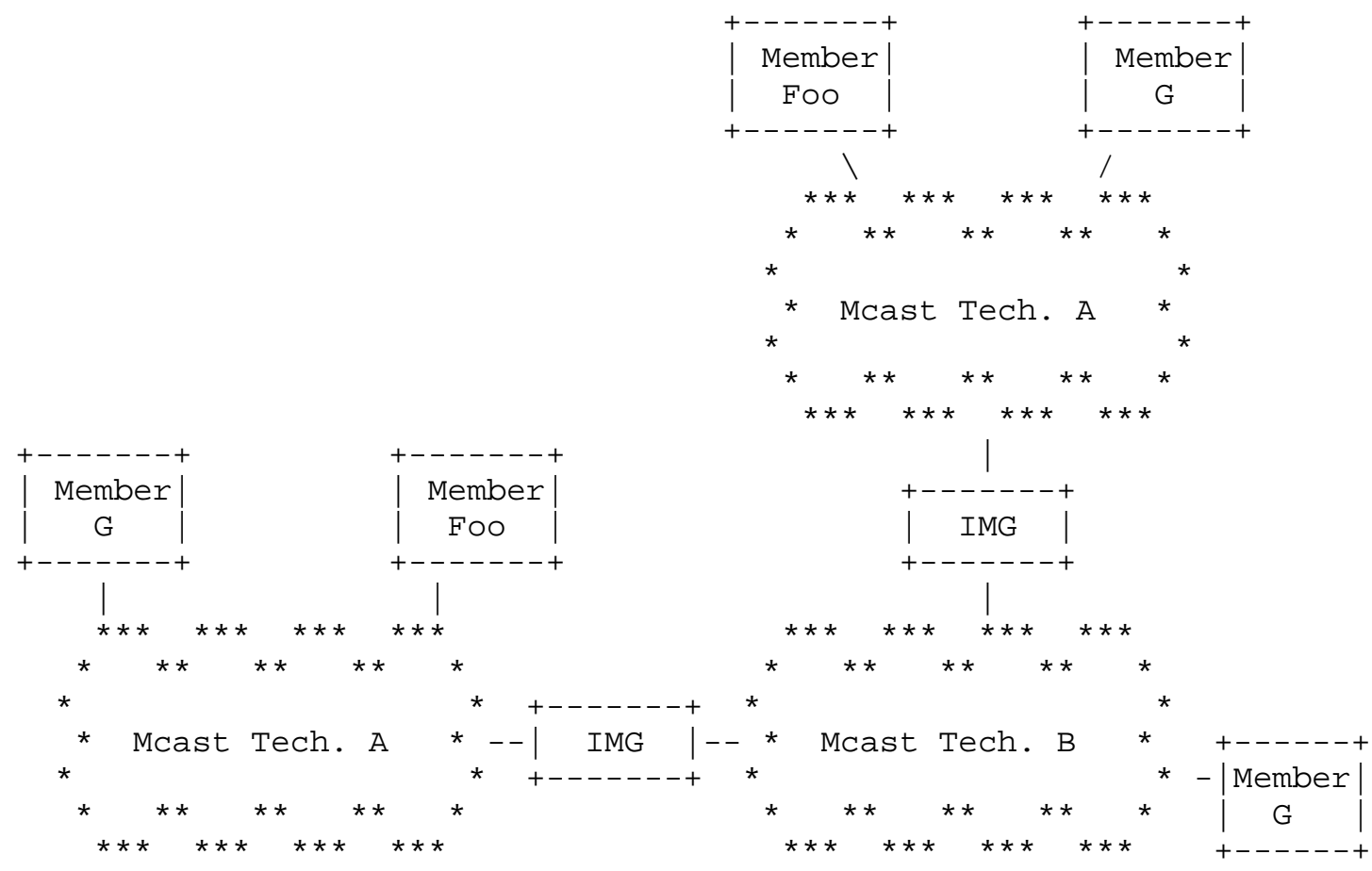

Figure 2: Reference Scenarios for Hybrid Multicast, Interconnecting Group Members from Isolated Homogeneous and Heterogeneous Domains

\subsection{Group Communication API and Protocol Stack}

The group communication API abstracts the socket concept and consists of four parts. Two parts combine the essential communication functions, while the remaining two offer optional extensions for enhanced monitoring and management:

Group Management Calls: provide the minimal API to instantiate an abstract multicast socket and manage group membership;

Send/Receive Calls: provide the minimal API to send and receive multicast data in a technology-transparent fashion;

Socket Options: provide extension calls for an explicit configuration of the multicast socket, such as setting hop limits or associated Interfaces;

Service Calls: provide extension calls that grant access to internal multicast states of an Interface, such as the Multicast Groups under subscription or the multicast forwarding information base. 
Multicast applications that use the common API require assistance from a group communication stack. This protocol stack serves two needs:

- It provides system-level support to transfer the abstract functions of the common API, including namespace support, into protocol operations at Interfaces.

- It provides group communication services across different multicast technologies at the local host.

A general initiation of a multicast communication in this setting proceeds as follows:

1. An application opens an abstract multicast socket.

2. The application subscribes to / leaves / (de)registers a group using a Group Name.

3. An intrinsic function of the stack maps the logical group ID (Group Name) to a technical group ID (Group Address). This function may make use of deployment-specific knowledge, such as available technologies and Group Address management in its domain.

4. Packet distribution proceeds to and from one or several multicast-enabled Interfaces.

The abstract multicast socket represents a group communication channel composed of one or multiple Interfaces. A socket may be created without explicit Interface association by the application, which leaves the choice of the underlying forwarding technology to the group communication stack. However, an application may also bind the socket to one or multiple dedicated Interfaces and therefore predefine the forwarding technology and the Multicast Namespace(s) of the Group Address (es).

Applications are not required to maintain mapping states for Group Addresses. The group communication stack accounts for the mapping of the Group Name to the Group Address(es) and vice versa. Multicast data passed to the application will be augmented by the corresponding Group Name. Multiple multicast subscriptions thus can be conducted on a single multicast socket without the need for Group Name encoding on the application side. 
Hosts may support several multicast protocols. The group communication stack discovers available multicast-enabled Interfaces. It provides a minimal hybrid function that bridges data between different Interfaces and Multicast Domains. The details of service discovery are out of scope for this document.

The extended multicast functions can be implemented by middleware, as conceptually presented in Figure 3 .
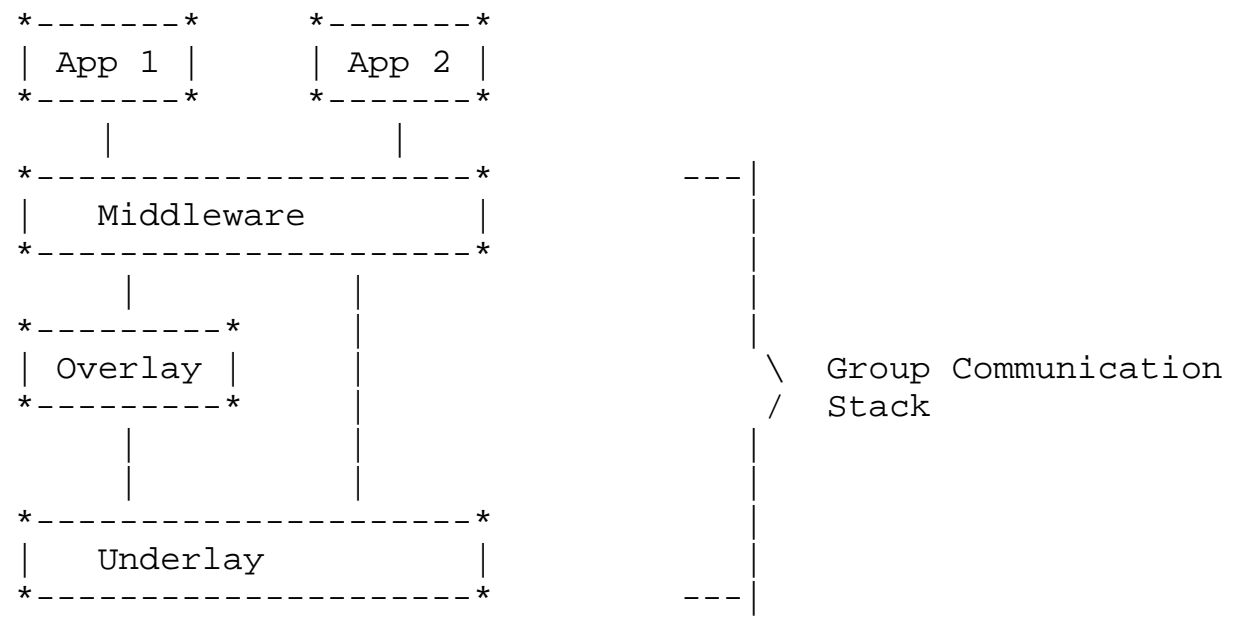

Figure 3: Architecture of a Group Communication Stack with Middleware Offering Uniform Access to Multicast in Underlay and Overlay

\subsection{Naming and Addressing}

Applications use Group Names to identify groups. Names can uniquely determine a group in a global communication context and hide technological deployment for data distribution from the application. In contrast, multicast forwarding operates on Group Addresses. Even though both identifiers may be symbolically identical, they carry different meanings. They may also belong to different Multicast Namespaces. The namespace of a Group Address reflects a routing technology, while the namespace of a Group Name represents the context in which the application operates.

URIs [RFC3986] are a common way to represent namespace-specific identifiers in applications in the form of an abstract metadata type. Throughout this document, all Group Names follow a URI notation using the syntax defined in Section 4.2. Examples are ham:ip:224.1.2.3:5000 for a canonical IPv4 ASM group at UDP port 5000 and ham:sip:news@cnn.com for application-specific naming with service instantiator and default port selection. 
An implementation of the group communication stack can provide convenience functions that detect the namespace of a Group Name or further optimize service instantiation. In practice, such a library would provide support for high-level data types to the application, similar to some versions of the current socket API (e.g., InetAddress in Java). Using this data type could implicitly determine the namespace. The details of automatic namespace identification or service handling are out of scope for this document.

\subsection{Namespaces}

Namespace identifiers in URIs are placed in the scheme element and characterize syntax and semantics of the group identifier. They enable the use of convenience functions and high-level data types while processing URIs. When used in names, they may indicate an application context or may facilitate a default mapping and a recovery of names from addresses. When used in addresses, they characterize the group identifier's type.

In compliance with the URI concept, namespace schemes can be added. Examples of schemes are generic (see Section 4.2.3) or inherited from applications (see Section 4.2.4).

\subsection{Name-to-Address Mapping}

The multicast communication paradigm requires all group members to subscribe to the same Group Name, taken from a common Multicast Namespace, and to thereby identify the group in a technology-agnostic way. Following this common API, a sender correspondingly registers a Group Name prior to transmission.

At communication end points, Group Names require a mapping to Group Addresses prior to service instantiation at the Interfaces of the end points. Similarly, a mapping is needed at gateways to consistently translate between Group Addresses from different namespaces. Two requirements need to be met by a mapping function that translates between Multicast Names and Addresses:

a. For a given Group Name, identify an Address that is appropriate for a local distribution instance.

b. For a given Group Address, invert the mapping to recover the Group Name.

In general, mappings can be complex and do not need to be invertible. A mapping can be realized by embedding smaller namespaces into larger namespaces or selecting an arbitrary, unused ID in a smaller target namespace. For example, it is not obvious how to map a large 
identifier space (e.g., IPv6) to a smaller, collision-prone set like IPv4 (see [MCAST-v4v6-FRAMEWORK], [MCAST-V4v6], and [RFC6219]). Mapping functions can be stateless in some contexts but may require states in others. The application of such functions depends on the cardinality of the namespaces, the structure of address spaces, and possible address collisions. However, some namespaces facilitate a canonical, invertible transformation to default address spaces.

\subsubsection{Canonical Mapping}

Some Multicast Namespaces defined in Section 3.4 can express a canonical default mapping. For example, ham:ip:224.1.2.3:5000 indicates the correspondence to 224.1.2.3 in the default IPv4 multicast address space at port 5000. This default mapping is bound to a technology and may not always be applicable, e.g., in the case of address collisions. Note that under canonical mapping, the multicast URI can be completely recovered from any data message received within this group.

\subsubsection{Mapping at End Points}

Multicast listeners or senders require a name-to-address conversion for all technologies they actively run in a group. Even though a mapping applies to the local Multicast Domain only, end points may need to learn a valid Group Address from neighboring nodes, e.g., from a gateway in the collision-prone IPv4 domain. Once set, an end point will always be aware of the name-to-address correspondence and thus can autonomously invert the mapping.

\subsubsection{Mapping at Inter-Domain Multicast Gateways}

Multicast data may arrive at an IMG via one technology and request that the gateway re-address packets for another distribution system. At initial arrival, the IMG may not have explicit knowledge of the corresponding Multicast Group Name. To perform a consistent mapping, the Group Name needs to be acquired. It may have been distributed at source registration or may have been learned from a neighboring node, the details of which are beyond the scope of this document.

\subsection{A Note on Explicit Multicast (Xcast)}

In Explicit Multicast (Xcast) [RFC5058], the multicast source explicitly predefines the receivers. From a conceptual perspective, Xcast is an additional distribution technology (i.e., a new technology-specific Interface) for this API. Xcast requires aggregated knowledge of receivers that is available at the origin of 
the distribution tree. The instantiation part of the Group Name may refer to such a management instance and tree root, which can be the source or some co-located processor.

An implementation of Xcast then requires a topology-dependent mapping of the Group Name to the set of subscribers. The defining details of this multi-destination mapping are out of scope for this document.

\subsection{MTU Handling}

This API considers a multi-technology scenario in which different technologies may have different Maximum Transmission Unit (MTU) sizes. Even if the MTU size between two hosts has been determined, it may change over time, as initiated by either the network (e.g., path changes) or end hosts (e.g., Interface changes due to mobility).

The design of this API is based on the objective of robust communication and easy application development. MTU handling and the implementation of fragmentation are thus guided by the following observations:

Application: Application programmers need a simple way to transmit packets in a technology-agnostic fashion. For this, it is convenient at the time of coding to rely on a transparent maximum amount of data that can be sent in one message from a socket. A regular program flow should not be distracted by querying and changing MTU sizes. Technically, the configuration of the maximum message size used by the application programmer may change and disrupt communication when (a) Interfaces are added or excluded or (b) the path MTU changes during transmission and thus disables the corresponding Interfaces.

Middleware: Middleware situated between application and technology Interfaces ensures a general packet-handling capability, which in turn prevents the application programmer from implementing fragmentation. A uniform maximum message size that cannot be changed during runtime shall be guaranteed by the group communication stack (e.g., middleware). Otherwise, this would conflict with a technology-agnostic application.

Technology Interfaces: Fragmentation requirements depend on the technology in use. Hence, the (technology-bound) Interfaces need to cope with MTU sizes that may vary among Interfaces and along different paths. 
The concept of this API also aims at guaranteeing a maximum message size for the application programmer, to thereby handle fragmentation at the Interface level, if needed. Nevertheless, the application programmer should be able to determine the technology-specific atomic message size to optimize data distribution, or for other reasons.

The uniform maximum message size should take realistic values (e.g., following IP clients) to enable smooth and efficient services. A detailed selection scheme of MTU values is out of scope for this document.

\section{Common Multicast API}

\subsection{Notation}

The following description of the common multicast API is expressed in pseudo-syntax. Variables that are passed to function calls are declared by "in", and return values are declared by "out". A list of elements is denoted by "<>". The pseudo-syntax assumes that lists include an attribute that represents the number of elements.

The corresponding $\mathrm{C}$ signatures are defined in Appendix A.

\subsection{URI Scheme Definition}

Multicast Names and Multicast Addresses used in this API are represented by a URI scheme that is specified in the following subsections. A corresponding ham-URI denotes a multicast channel and may be dereferenced to retrieve data published to that channel.

\subsubsection{Syntax}

The syntax of the multicast URI is specified using the Augmented Backus-Naur Form (ABNF) [RFC5234] and is defined as follows:

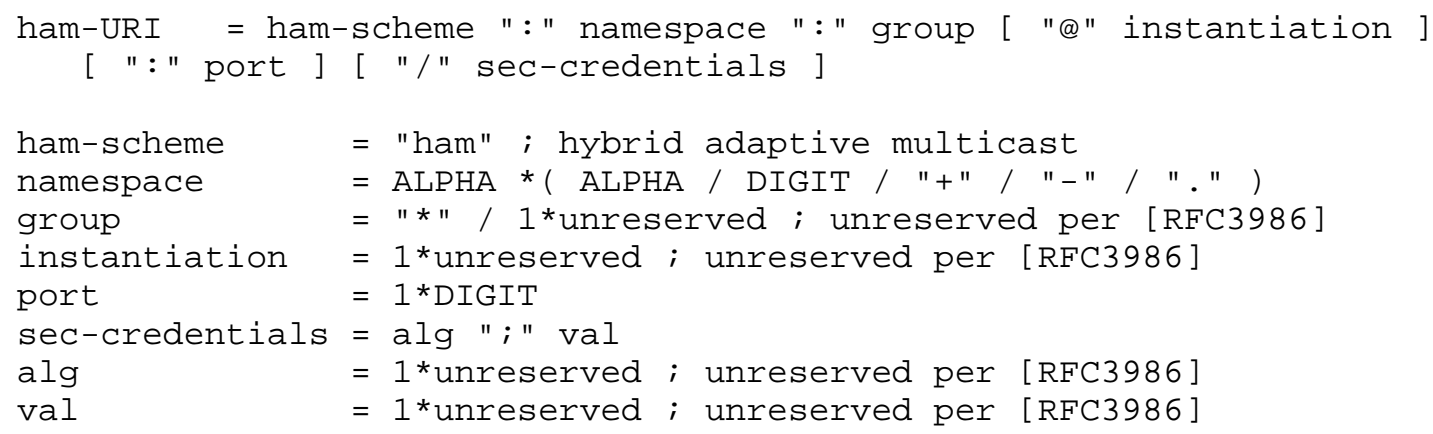


Percent-encoding is applied to distinguish between reserved and unreserved assignments of the same character in the same ham-URI component (cf. [RFC3986]).

\subsubsection{Semantic}

The semantic of the different parts of the URI is defined as follows:

ham-scheme: refers to the specification of the assigned identifier "ham".

namespace: takes the role of the Multicast Namespace. It defines the syntax of the group and instantiation part of the ham-URI. A basic syntax for these elements is specified in section 4.2.1. The namespace may further restrict the syntax of designators. Example namespaces are described in sections 4.2.3 and 4.2.4.

group: uniquely identifies the group within the Multicast Namespace given in the namespace. The literal "*" represents all members of the Multicast Group.

instantiation: identifies the entity that generates the instance of the group (e.g., a SIP domain or a source in SSM, a dedicated routing entity, or a named processor that accounts for the group communication), using syntax and semantics as defined by the namespace. This parameter is optional. Note that ambiguities (e.g., identical node addresses in multiple overlay instances) can be distinguished by ports.

port: identifies a specific application at an instance of a group. This parameter is optional.

sec-credentials: used to implement security mechanisms (e.g., to authorize Multicast Group access or authenticate multicast operations). This parameter is optional. "alg" represents the security algorithm in use. "val" represents the actual value for Authentication, Authorization, and Accounting (AAA). Note that security credentials may carry a distinct technical meaning w.r.t. AAA schemes and may differ between group members. Hence, the sec-credentials are not considered part of the Group Name. 


\subsubsection{Generic Namespaces}

IP: This namespace is comprised of regular IP node naming, i.e., DNS names and addresses taken from any version of the Internet Protocol. The syntax of the group and instantiation follows the "host" definition in [RFC3986], Section 3.2.2. A processor dealing with the IP namespace is required to determine the syntax (DNS name, IP address, version) of the group and instantiation expression.

SHA-2: This namespace carries address strings compliant with SHA-2 hash digests. The syntax of the group and instantiation follows the "val" definition in [RFC6920], Section 3. A processor handling those strings is required to determine the length of the expressions and passes appropriate values directly to a corresponding overlay.

Opaque: This namespace transparently carries strings without further syntactical information, meanings, or associated resolution mechanisms. The corresponding syntax for the group and instantiation part of the ham-URI is defined in section 4.2.1.

\subsubsection{Application-Centric Namespaces}

SIP: The SIP namespace is an example of an application-layer scheme that bears inherent group functions (conferencing). SIP conference URIs may be directly exchanged and interpreted at the application, and mapped to Group Addresses at the system level to generate a corresponding Multicast Group. The syntax of the group and instantiation is represented by the "userinfo" component [RFC3261], Section 25.1.

RELOAD: This namespace covers address strings that are valid in a REsource LOcation And Discovery [RELOAD] overlay network. A processor handling those strings may pass these values directly to a corresponding overlay that may manage multicast distribution according to [RFC7019].

\subsubsection{Future Namespaces}

The concept of the common multicast API allows for any namespace that complies with the superset syntax defined in Section 4.2.1. This document specifies a basic set of Multicast Namespaces in Sections 4.2.3 and 4.2.4. If additional namespaces are needed in the future, a registry for those namespaces should be created and should be defined in a future document. All namespaces defined in such a document should then also be assigned to the registry. 


\subsection{Additional Abstract Data Types}

\subsubsection{Interface}

The Interface denotes the layer and instance on which the corresponding call takes effect. In agreement with [RFC3493], we identify an Interface by an identifier, which is a positive integer starting at 1 .

Properties of an Interface are stored in the following data structure:

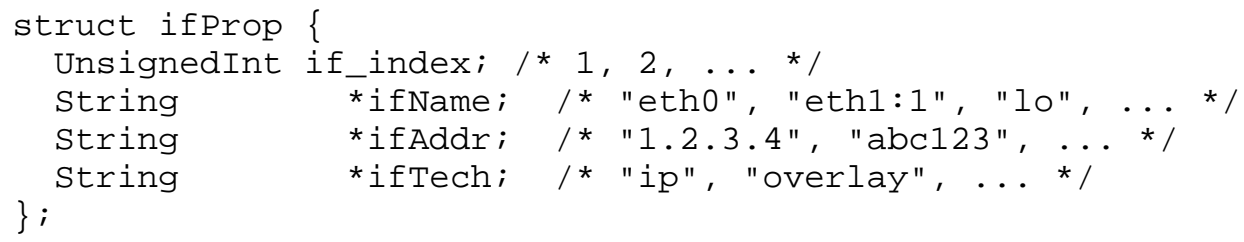

The following function retrieves all available Interfaces from the system:

getInterfaces (out Interface <ifs>);

It extends the functions for Interface identification as defined in [RFC3493], Section 4 and can be implemented by:

struct ifProp (out IfProp <ifsProps>);

\subsubsection{Membership Events}

A membership event is triggered by a multicast state change that is observed by the current node. It is related to a specific Group Name and may be receiver or source oriented.

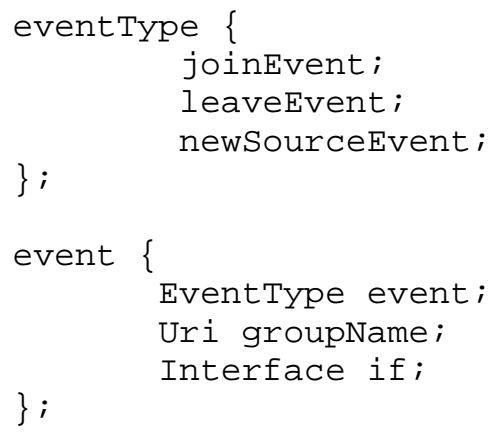


An event will be created by the group communication stack and passed to applications that have registered for events.

4.4. Group Management Calls

4.4.1. Create

The create call initiates a multicast socket and provides the application programmer with a corresponding handle. If no Interfaces will be assigned based on the call, the default Interface will be selected and associated with the socket. The call returns an error code in the case of failures, e.g., due to non-operational communication middleware.

createMSocket (in Interface <ifs>, out socket s);

The ifs argument denotes a list of Interfaces (if_indexes) that will be associated with the multicast socket. This parameter is optional.

On success, a multicast socket identifier is returned; otherwise, it is NULL.

4.4.2. Delete

The delete call removes the multicast socket. deleteMSocket (in socket s, out Int error);

The s argument identifies the multicast socket for destruction.

On success, the out parameter error is 0 ; otherwise, -1 is returned.

\subsubsection{Join}

The join call initiates a subscription for the given Group Name. Depending on the Interfaces that are associated with the socket, this may result in an IGMP / Multicast Listener Discovery (MLD) report or overlay subscription, for example. join(in Socket s, in Uri groupName, out Int error);

The s argument identifies the multicast socket.

The groupName argument identifies the group.

On success, the out parameter error is 0 ; otherwise, -1 is returned. 


\subsubsection{Leave}

The leave call results in an unsubscription for the given Group Name. leave(in socket $\mathrm{s}$, in Uri groupName, out Int error);

The s argument identifies the multicast socket.

The groupName argument identifies the group.

On success, the out parameter error is 0 ; otherwise, -1 is returned.

\subsubsection{Source Register}

The srcRegister call registers a source for a group on all active Interfaces of the socket $\mathrm{s}$. This call may assist group distribution in some technologies -- for example, the creation of sub-overlays -or may facilitate a name-to-address mapping. Likewise, it may remain without effect in some multicast technologies. srcRegister(in Socket $s$, in Uri groupName, out Interface <ifs>, out Int error);

The s argument identifies the multicast socket.

The groupName argument identifies the Multicast Group to which a source intends to send data.

The ifs argument points to the list of Interface indexes for which the source registration failed. A NULL pointer is returned if the list is empty. This parameter is optional.

If source registration succeeded for all Interfaces associated with the socket, the out parameter error is 0 ; otherwise, -1 is returned.

\subsubsection{Source Deregister}

The srcDeregister call indicates that a source no longer intends to send data to the Multicast Group. This call may remain without effect in some multicast technologies.

srcDeregister(in Socket $s$, in Uri groupName, out Interface $\langle$ ifs $\rangle$, out Int error);

The s argument identifies the multicast socket.

The groupName argument identifies the Multicast Group to which a source has stopped sending multicast data. 
The ifs argument points to the list of Interfaces for which the source deregistration failed. A NULL pointer is returned if the list is empty.

If source deregistration succeeded for all Interfaces associated with the socket, the out parameter error is 0 ; otherwise, -1 is returned.

4.5. Send and Receive Calls

4.5.1. Send

The send call passes multicast data destined for a Multicast Name from the application to the multicast socket.

It is worth noting that it is the choice of the programmer to send data via one socket per group or to use a single socket for multiple groups.

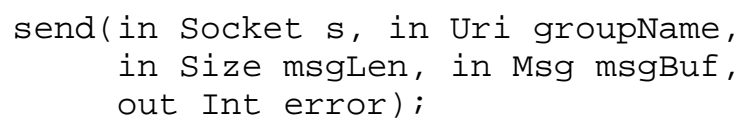

The s argument identifies the multicast socket.

The groupName argument identifies the group to which data will be sent.

The msgLen argument holds the length of the message to be sent.

The msgBuf argument passes the multicast data to the multicast socket.

On success, the out parameter error is 0 ; otherwise, -1 is returned. A message that is too long is indicated by an implementation-specific error code (e.g., EMSGSIZE in C).

\subsubsection{Receive}

The receive call passes multicast data and the corresponding Group Name to the application. This may come in a blocking or non-blocking variant.

It is worth noting that it is the choice of the programmer to receive data via one socket per group or to use a single socket for multiple groups. 


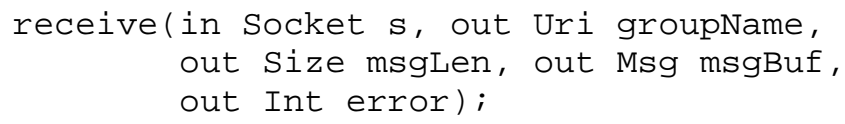

The s argument identifies the multicast socket.

The groupName argument identifies the Multicast Group for which data was received.

The msgLen argument holds the length of the received message.

The msgBuf argument points to the payload of the received multicast data.

On success, the out parameter error is 0 ; otherwise, -1 is returned. A message that is too long is indicated by an implementation-specific error code (e.g., EMSGSIZE).

4.6. Socket Options

The following calls configure an existing multicast socket.

4.6.1. Get Interfaces

The getInterfaces call returns an array of all available multicast communication Interfaces associated with the multicast socket. getInterfaces (in socket $s$, out Interface <ifs>, out Int error);

The s argument identifies the multicast socket.

The ifs argument points to an array of Interface index identifiers.

On success, the out parameter error is 0 ; otherwise, -1 is returned.

4.6.2. Add Interface

The addInterface call adds a distribution channel to the socket. This may be an overlay or underlay Interface, e.g., IPv6 or Distributed Hash Table (DHT). Multiple Interfaces of the same technology may be associated with the socket.

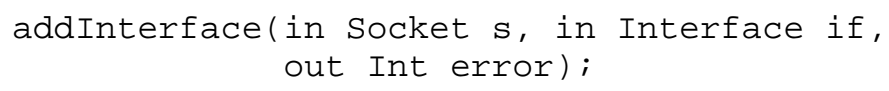


The $s$ and if arguments identify a multicast socket and Interface, respectively.

On success, the value 0 is returned; otherwise, -1 is returned.

\subsubsection{Delete Interface}

The delinterface call removes the Interface from the multicast socket.

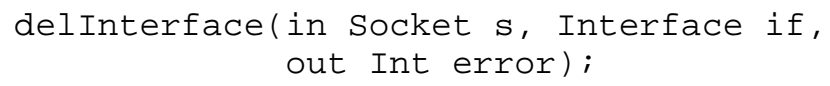

The $s$ and if arguments identify a multicast socket and Interface, respectively.

On success, the out parameter error is 0 ; otherwise, -1 is returned.

4.6.4. Set TTL

The setTTL call configures the maximum hop count for the socket that a multicast message is allowed to traverse.

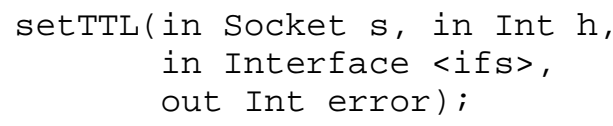

The $\mathrm{s}$ and h arguments identify a multicast socket and the maximum hop count, respectively.

The ifs argument points to an array of Interface index identifiers. This parameter is optional.

On success, the out parameter error is 0 ; otherwise, -1 is returned.

4.6.5. Get TTL

The getTTL call returns the maximum hop count that a multicast message is allowed to traverse for the interface bound to the socket.

The s argument identifies a multicast socket.

The if argument identifies an interface that is bound to socket $\mathrm{s}$. 
The h argument holds the maximum number of hops associated with the interface.

On success, the out parameter error is 0 ; otherwise, -1 is returned.

4.6.6. Atomic Message Size

The getAtomicMsgSize function returns the maximum message size that an application is allowed to transmit per socket at once without fragmentation. This value depends on the Interfaces associated with the socket in use and thus may change during runtime.

getAtomicMsgSize(in socket s, out Int return);

On success, the function returns a positive value of appropriate message size; otherwise, -1 is returned.

4.7. Service Calls

4.7.1. Group set

The groupset call returns all Multicast Groups registered at a given Interface. This information can be provided by group management states or routing protocols. The return values distinguish between sender and listener states.

struct Groupset \{

Uri groupName; / registered Multicast Group * /

Int type; $\quad / * 0=$ listener state, 1 = sender state, 2 = sender and listener state */

\}

groupset (in Interface if, out Groupset <groupset>, out Int error);

The if argument identifies the Interface for which states are maintained.

The groupset argument points to a list of group states.

On success, the out parameter error is 0 ; otherwise, -1 is returned. 


\subsubsection{Neighbor Set}

The neighborset function returns the set of neighboring nodes for a given Interface as seen by the multicast routing protocol.

$$
\text { neighborSet (in Interface if, }
$$

out Uri <neighborsAddresses>, out Int error);

The if argument identifies the Interface for which information regarding neighbors is requested.

The neighborsAddresses argument points to a list of neighboring nodes on a successful return.

On success, the out parameter error is 0 ; otherwise, -1 is returned.

\subsubsection{Children set}

The childrenset function returns the set of child nodes that receive multicast data from a specified Interface for a given group. For a common multicast router, this call retrieves the multicast forwarding information base per Interface.

childrenset(in Interface if, in Uri groupName, out Uri <childrenAddresses>, out Int error);

The if argument identifies the Interface for which information regarding children is requested.

The groupName argument defines the Multicast Group for which distribution is considered.

The childrenAddresses argument points to a list of neighboring nodes on a successful return.

On success, the out parameter error is 0 ; otherwise, -1 is returned.

\subsubsection{Parent set}

The parentSet function returns the set of neighbors from which the current node receives multicast data at a given Interface for the specified group.

parentSet(in Interface if, in Uri groupName, out Uri <parentsAddresses>, out Int error);

The if argument identifies the Interface for which information regarding parents is requested. 
The groupName argument defines the Multicast Group for which distribution is considered.

The parentsAddresses argument points to a list of neighboring nodes on a successful return.

On success, the out parameter error is 0 ; otherwise, -1 is returned.

\subsubsection{Designated Host}

The designatedHost function inquires about whether this host has the role of a designated forwarder (or querier), or not. Such information is provided by almost all multicast protocols to prevent packet duplication, if multiple multicast instances provide service on the same subnet.

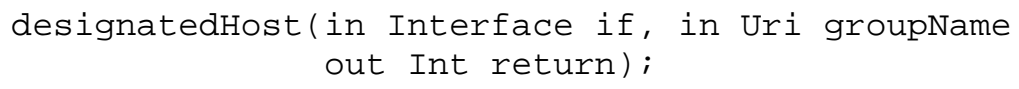

The if argument identifies the Interface for which information regarding designated forwarding is requested.

The groupName argument specifies the group for which the host may attain the role of designated forwarder.

The function returns 1 if the host is a designated forwarder or querier. The return value -1 indicates an error. Otherwise, 0 is returned.

\subsubsection{Enable Membership Events}

The enableEvents function registers an application at the group communication stack to receive information about group changes. State changes are the result of new receiver subscriptions or leaves, as well as source changes. Upon receiving an event, the group service may obtain additional information from further service calls. enableEvents ();

Calling this function, the stack starts to pass membership events to the application. Each event includes an event type identifier and a Group Name (cf. Section 4.3.2).

The multicast protocol does not have to support membership tracking in order to enable this feature. This function can also be implemented at the middleware layer. 


\subsubsection{Disable Membership Events}

The disableEvents function deactivates the information about group state changes.

$$
\text { disableEvents (); }
$$

On success, the stack will not pass membership events to the application.

4.7.8. Maximum Message Size

The getMaxMsgSize function returns the maximum message size that an application is allowed to transmit per socket at once. This value is statically guaranteed by the group communication stack.

getMaxMsgSize(out Int return);

On success, the function returns a positive value of allowed message size; otherwise, -1 is returned.

5. Implementation

A reference implementation of the Common API for Transparent Hybrid Multicast is available with the HAMcast stack [HAMcast-DEV] [GC2010] [LCN2012]. This open-source software supports the multicast API (C++ and Java library) for group application development, the middleware as a user space system service, and several multicast-technology modules. The middleware is implemented in $\mathrm{C}++$.

This API is verified and adjusted based on the real-world experiences gathered in the HAMcast project, and by additional users of the stack.

6. IANA Considerations

This document specifies the "ham" URI scheme that has been registered by IANA as one of the "Provisional URI Schemes" according to [RFC4395].

URI scheme name ham

Status provisional

URI scheme syntax See section 4.2.1.

URI scheme semantics see section 4.2.2.

Waehlisch, et al. Experimental [Page 30] 


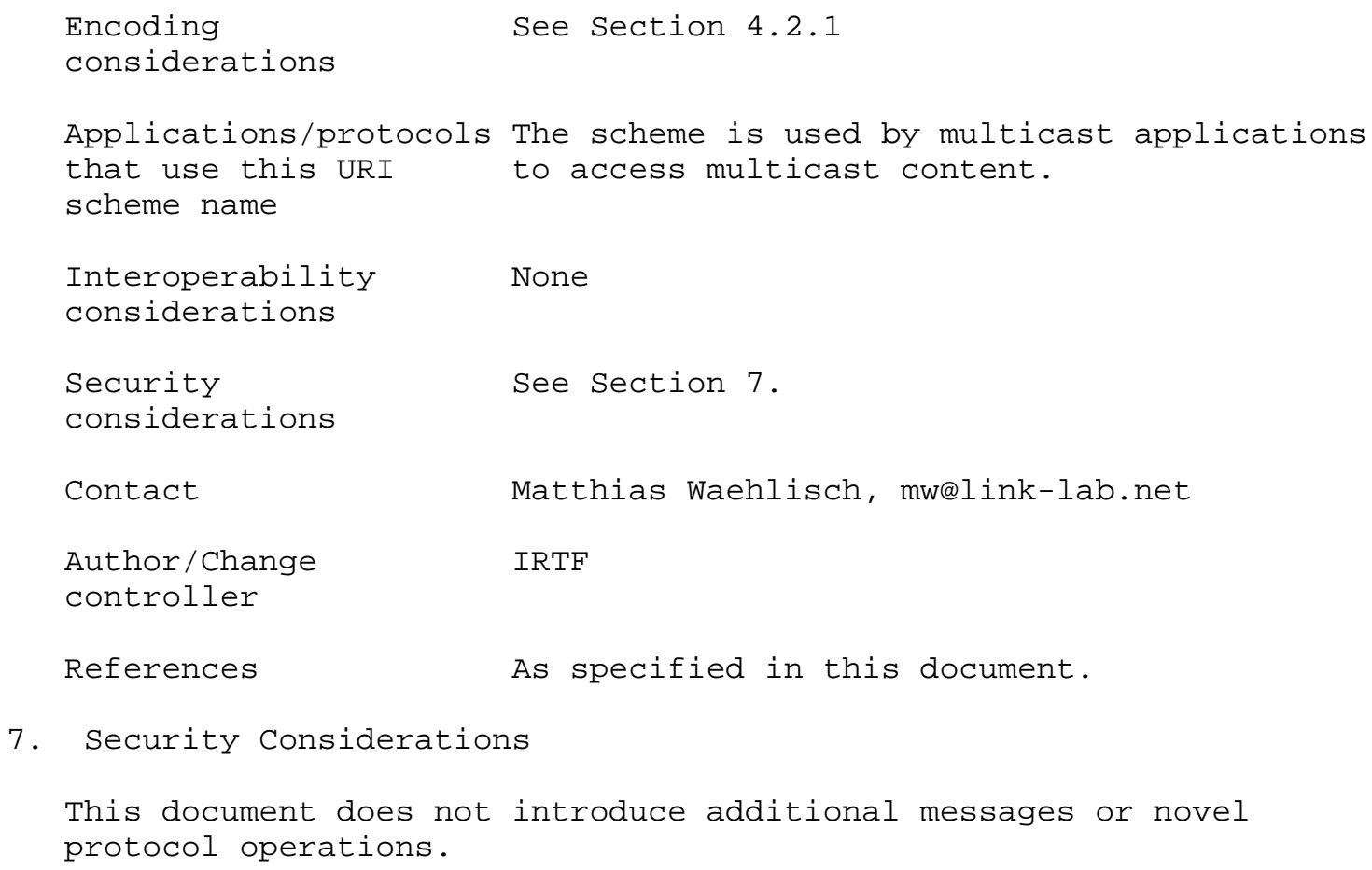

8. Acknowledgements

We would like to thank the HAMcast team at the HAW Hamburg -- Nora Berg, Gabriel Hege, Fabian Holler, Alexander Knauf, Sebastian Meiling, Sebastian Woelke, and Sebastian Zagaria -- for many fruitful discussions and for their continuous critical feedback while implementing the common multicast API and hybrid multicast middleware. Special thanks to Dominik Charousset of the HAMcast team for in-depth perspectives on the matter of code. We gratefully acknowledge WeeSan, Mario Kolberg, and John Buford for reviewing and their suggestions to improve the document. We would like to thank the Name-Based Socket BoF (in particular Dave Thaler) for clarifying insights into the question of meta-function calls. We thank Lisandro Zambenedetti Granville and Tony Li for very careful reviews of the pre-final versions of this document. Barry Leiba and Graham Klyne provided very constructive input to find a suitable URI scheme. They are gratefully acknowledged.

This work is partially supported by the German Federal Ministry of Education and Research within the HAMcast project (see <http://hamcast.realmv6.org>), which is part of G-Lab. 


\section{References}

\subsection{Normative References}

[RFC1075] Waitzman, D., Partridge, C., and S. Deering, "Distance Vector Multicast Routing Protocol", RFC 1075, November 1988 .

[RFC2710] Deering, S., Fenner, W., and B. Haberman, "Multicast Listener Discovery (MLD) for IPv6", RFC 2710, October 1999 .

[RFC3261] Rosenberg, J., Schulzrinne, H., Camarillo, G., Johnston, A., Peterson, J., Sparks, R., Handley, M., and E. Schooler, "SIP: Session Initiation Protocol", RFC 3261, June 2002 .

[RFC3376] Cain, B., Deering, S., Kouvelas, I., Fenner, B., and A. Thyagarajan, "Internet Group Management Protocol, Version 3", RFC 3376, October 2002 .

[RFC3493] Gilligan, R., Thomson, S., Bound, J., McCann, J., and W. Stevens, "Basic Socket Interface Extensions for IPv6", RFC 3493, February 2003.

[RFC3678] Thaler, D., Fenner, B., and B. Quinn, "Socket Interface Extensions for Multicast Source Filters", RFC 3678, January 2004 .

[RFC3810] Vida, R. and L. Costa, "Multicast Listener Discovery Version 2 (MLDv2) for IPv6", RFC 3810, June 2004.

[RFC3986] Berners-Lee, T., Fielding, R., and L. Masinter, "Uniform Resource Identifier (URI): Generic Syntax", STD 66, RFC 3986, January 2005 .

[RFC4395] Hansen, T., Hardie, T., and L. Masinter, "Guidelines and Registration Procedures for New URI Schemes", BCP 35, RFC 4395, February 2006.

[RFC4601] Fenner, B., Handley, M., Holbrook, H., and I. Kouvelas, "Protocol Independent Multicast - Sparse Mode (PIM-SM) : Protocol Specification (Revised)", RFC 4601, August 2006.

[RFC4604] Holbrook, H., Cain, B., and B. Haberman, "Using Internet Group Management Protocol Version 3 (IGMPv3) and Multicast Listener Discovery Protocol Version 2 (MLDv2) for SourceSpecific Multicast", RFC 4604, August 2006. 
[RFC5015] Handley, M., Kouvelas, I., Speakman, T., and L. Vicisano, "Bidirectional Protocol Independent Multicast

(BIDIR-PIM)", RFC 5015, October 2007.

[RFC5058] Boivie, R., Feldman, N., Imai, Y., Livens, W., and D. Ooms, "Explicit Multicast (Xcast) Concepts and Options", RFC 5058, November 2007.

[RFC5234] Crocker, D. and P. Overell, "Augmented BNF for Syntax Specifications: ABNF", STD 68, RFC 5234, January 2008.

[RFC6920] Farrell, S., Kutscher, D., Dannewitz, C., Ohlman, B., Keranen, A., and P. Hallam-Baker, "Naming Things with Hashes", RFC 6920, April 2013.

9.2. Informative References

[AMT] Bumgardner, G., "Automatic Multicast Tunneling", Work in Progress, October 2013.

[GC2010] Meiling, S., Charousset, D., Schmidt, T., and M. Waehlisch, "System-assisted Service Evolution for a Future Internet - The HAMcast Approach to Pervasive Multicast", Proc. IEEE GLOBECOM 2010 Workshops, MCS 2010, pp. 913-917, Piscataway, NJ, USA, IEEE Press, December 2010.

[HAMcast-DEV]

"HAMcast developers",

<http://hamcast.realmv6.org/developers>.

[LCN2012] Meiling, S., Schmidt, T., and M. Waehlisch, "Large-Scale Measurement and Analysis of One-Way Delay in Hybrid Multicast Networks", Proc. 37th Annual IEEE Conference on Local Computer Networks (LCN 2012), Piscataway, NJ, USA, IEEE Press, October 2012 .

[MCAST-V4v6]

Venaas, S., Asaeda, H., SUZUKI, S., and T. Fujisaki, "An IPv4 - IPv6 multicast translator", Work in Progress, December 2010 .

[MCAST-V4v6-FRAMEWORK] Venaas, S., Li, X., and C. Bao, "Framework for IPv4/IPv6 Multicast Translation", Work in Progress, June 2011.

[RELOAD] Jennings, C., Lowekamp, B., Ed., Rescorla, E., Baset, S., and H. Schulzrinne, "REsource LOcation And Discovery (RELOAD) Base Protocol", Work in Progress, February 2013. 
[RFC5757] Schmidt, T., Waehlisch, M., and G. Fairhurst, "Multicast Mobility in Mobile IP Version 6 (MIPv6): Problem statement and Brief Survey", RFC 5757, February 2010.

[RFC6219] Li, X., Bao, C., Chen, M., Zhang, H., and J. Wu, "The China Education and Research Network (CERNET) IVI Translation Design and Deployment for the IPv4/IPv6 Coexistence and Transition", RFC 6219, May 2011.

[RFC7019] Buford, J. and M. Kolberg, "Application-Layer Multicast Extensions to REsource LOcation And Discovery (RELOAD)", RFC 7019, September 2013.

[SIP-RELOAD ]

Jennings, C., Lowekamp, B., Rescorla, E., Baset, S., Schulzrinne, H., and T. Schmidt, Ed., "A SIP Usage for RELOAD", Work in Progress, July 2013. 
Appendix A. C Signatures

This section describes the C signatures of the common multicast API (Section 4).

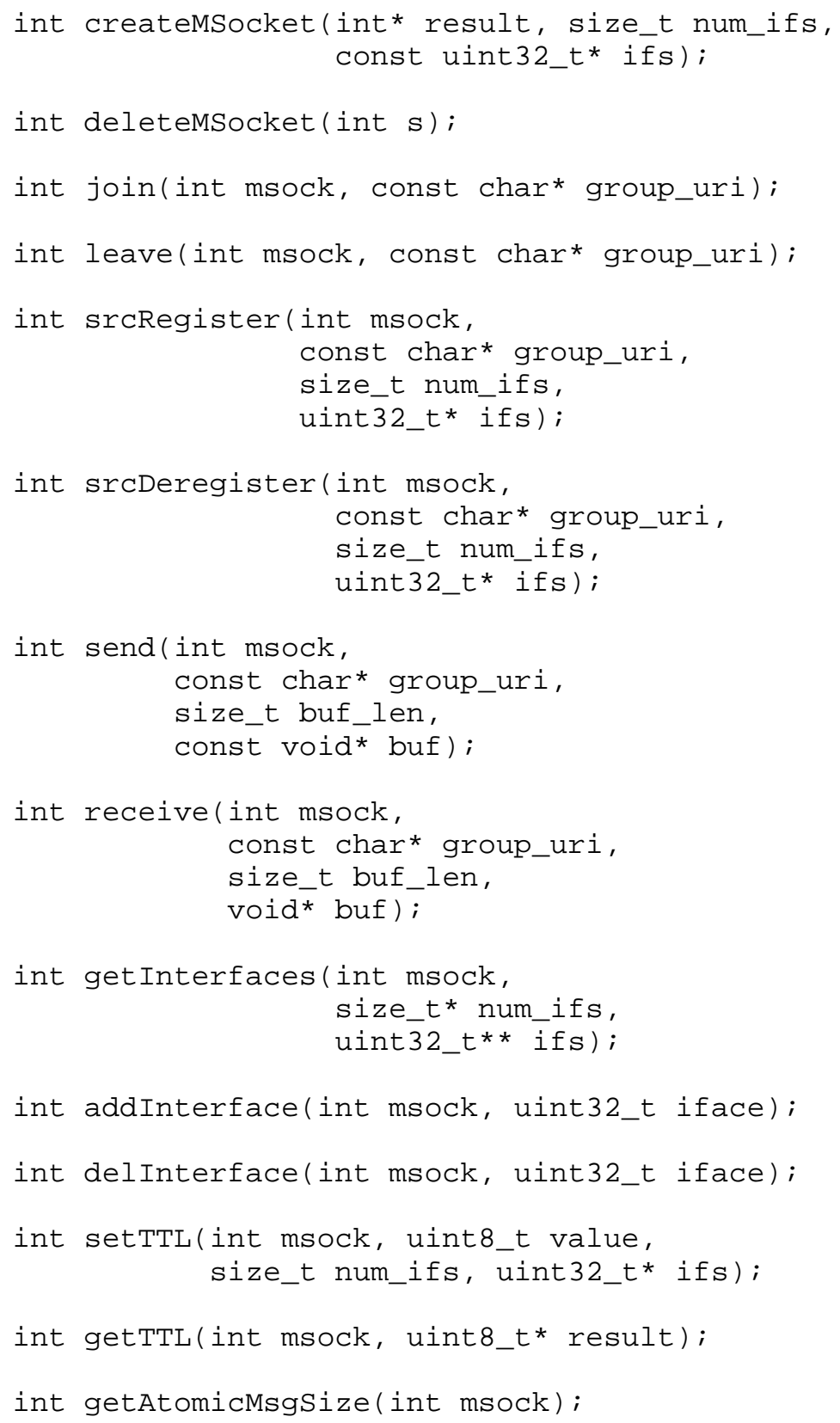




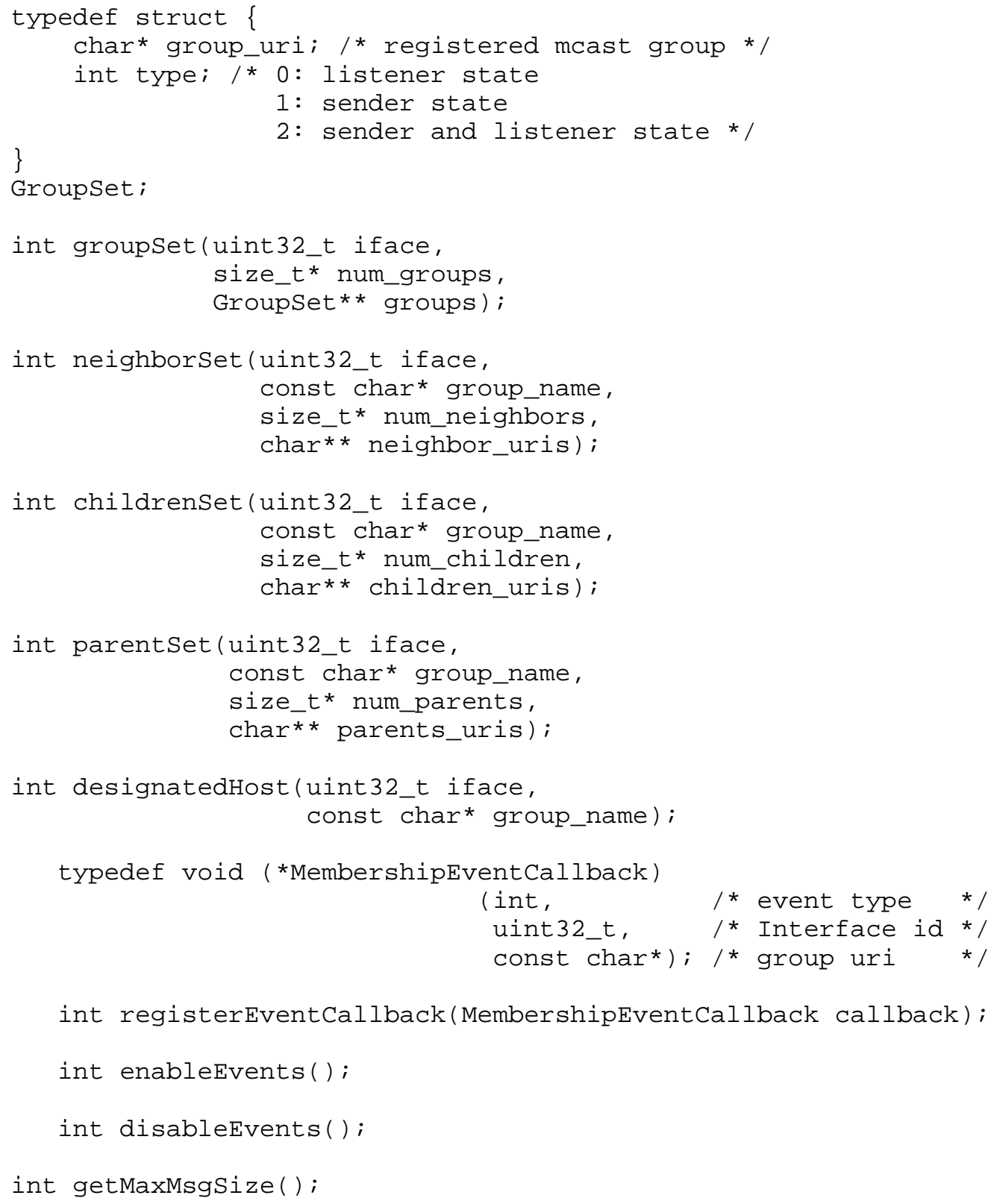


Appendix B. Use Case for the API

For the sake of readability, we demonstrate development of applications using the API based on a high-level Java-like syntax; we do not consider error handling.

-- Application above middleware:

//Initialize multicast socket;

//the middleware selects all available Interfaces

MulticastSocket $m=$ new MulticastSocket();

m.join (URI ("ham:ip:224.1.2.3:5000"));

m. join (URI ("ham:ip: [ff02:0:0:0:0:0:0:3]:6000"));

m. join (URI ("ham:sip:news@cnn.com")) ;

-- Middleware:

join(URI mcAddress) \{

//Select Interfaces in use

for all this.interfaces \{

switch (interface.type) \{

case "ipv6":

//... map logical ID to routing address

Inet6Address rtAddressIPv6 = new Inet6Address();

mapNametoAddress (mcAddress, rtAddressIPv6);

interface.join (rtAddressIPv6);

case "ipv4":

//... map logical ID to routing address

Inet4Address rtAddressIPv4 = new Inet4Address();

mapNametoAddress (mcAddress, rtAddressIPv4);

interface.join (rtAddressIPv4);

case "sip-session":

//... map logical ID to routing address

SIPAddress rtAddressSIP = new SIPAddress();

mapNametoAddress (mcAddress, rtAddressSIP) ;

interface.join (rtAddressSIP);

case "dht":

//... map logical ID to routing address

DHTAddress rtAddressDHT = new DHTAddress ();

mapNametoAddress (mcAddress, rtAddressDHT);

interface.join (rtAddressDHT);

/ / . .

\}

\}

Waehlisch, et al.

Experimental

[Page 37] 
Appendix C. Deployment Use Cases for Hybrid Multicast

This section describes the application of the defined API to implement an IMG.

\section{C.1. DVMRP}

The following procedure describes a transparent mapping of a DVMRP-based any-source multicast service to another many-to-many multicast technology, e.g., an overlay.

An arbitrary Distance Vector Multicast Routing Protocol (DVMRP) [RFC1075] router will not be informed of new receivers but will learn about new sources immediately. The concept of DVMRP does not provide any central multicast instance. Thus, the IMG can be placed anywhere inside the multicast region, but the IMG requires a DVMRP neighbor connectivity. Thus, the group communication stack used by the IMG is enhanced by a DVMRP implementation. New sources in the underlay will be advertised based on the DVMRP flooding mechanism and received by the IMG. Based on this, the event "new_source_event" is created and passed to the application. The relay agent initiates a corresponding join in the native network and forwards the received source data towards the overlay routing protocol. Depending on the group states, the data will be distributed to overlay peers.

DVMRP establishes source-specific multicast trees. Therefore, a graft message is only visible to DVMRP routers on the path from the new receiver subnet to the source, but in general not to an IMG. To overcome this problem, data of multicast senders in the overlay may become noticeable via the source Register call, as well as by an IMG that initiates an all-group join in the overlay using the namespace extension of the API. Each IMG is initially required to forward the data received in the overlay to the underlay, independent of native multicast receivers. Subsequent prunes may limit unwanted data distribution thereafter.

\section{C.2. PIM-SM}

The following procedure describes a transparent mapping of a PIM-SM-based any-source multicast service to another many-to-many multicast technology, e.g., an overlay.

The Protocol Independent Multicast - Sparse Mode (PIM-SM) [RFC4601] establishes rendezvous points (RPS). These entities receive listener subscriptions and source registering of a domain. For a continuous update, an IMG has to be co-located with an RP. Whenever PIM register messages are received, the IMG must signal internally a new multicast source using the event "new_source_event". Subsequently, 
the IMG joins the group and a shared tree between the RP and the sources will be established; this shared tree may change to a sourcespecific tree after PIM switches to phase three. Source traffic will be forwarded to the RP based on the IMG join, even if there are no further receivers in the native Multicast Domain. Designated routers of a PIM domain send receiver subscriptions towards the PIM-SM RP. The reception of such messages initiates the event "join_event" at the IMG, which initiates a join towards the overlay routing protocol. Overlay multicast data arriving at the IMG will then be transparently forwarded in the underlay network and distributed through the RP instance.

C. 3. PIM-SSM

The following procedure describes a transparent mapping of a PIM-SSM-based source-specific multicast service to another one-to-many multicast technology, e.g., an overlay.

PIM Source-Specific Multicast (PIM-SSM) is defined as part of PIM-SM and admits source-specific joins $(S, G)$ according to the sourcespecific host group model [RFC4604]. A multicast distribution tree can be established without the assistance of a rendezvous point.

Sources are not advertised within a PIM-SSM domain. Consequently, an IMG cannot anticipate the local join inside a sender domain and deliver a priori the multicast data to the overlay instance. If an IMG of a receiver domain initiates a group subscription via the overlay routing protocol, relaying multicast data fails, as data is not available at the overlay instance. The IMG instance of the receiver domain thus has to locate the IMG instance of the source domain to trigger the corresponding join. In agreement with the objectives of PIM-SSM, the signaling should not be flooded in the underlay and overlay.

A solution can be to intercept the subscription at both source sites and receiver sites: To monitor multicast receiver subscriptions ("join_event" or "leave_event") in the underlay, the IMG is placed on the path towards the source, e.g., at a domain border router. This router intercepts join messages and extracts the unicast source address $S$, initializing an IMG-specific join to $S$ via regular unicast. Multicast data arriving at the IMG of the sender domain can be distributed via the overlay. Discovering the IMG of a multicast sender domain may be implemented analogously to Automatic Multicast Tunneling [AMT] by anycast. Consequently, the source address $S$ of the group $(S, G)$ should be built based on an anycast prefix. The corresponding IMG anycast address for a source domain is then derived from the prefix of $S$. 


\section{C.4. BIDIR-PIM}

The following procedure describes a transparent mapping of a BIDIR-PIM-based any-source multicast service to another many-to-many multicast technology, e.g., an overlay.

Bidirectional PIM [RFC5015] is a variant of PIM-SM. In contrast to PIM-SM, the protocol pre-establishes bidirectional shared trees per group, connecting multicast sources and receivers. The rendezvous points are virtualized in BIDIR-PIM as an address to identify on-tree directions (up and down). Routers with the best link towards the (virtualized) rendezvous point address are selected as designated forwarders for a link-local domain and represent the actual distribution tree. The IMG is to be placed at the RP link, where the rendezvous point address is located. As source data in either case will be transmitted to the RP link, the BIDIR-PIM instance of the IMG receives the data and can internally signal new senders towards the stack via the "new_source_event". The first receiver subscription for a new group within a BIDIR-PIM domain needs to be transmitted to the RP to establish the first branching point. Using the "join_event", an IMG will thereby be informed of group requests from its domain, which are then delegated to the overlay. 


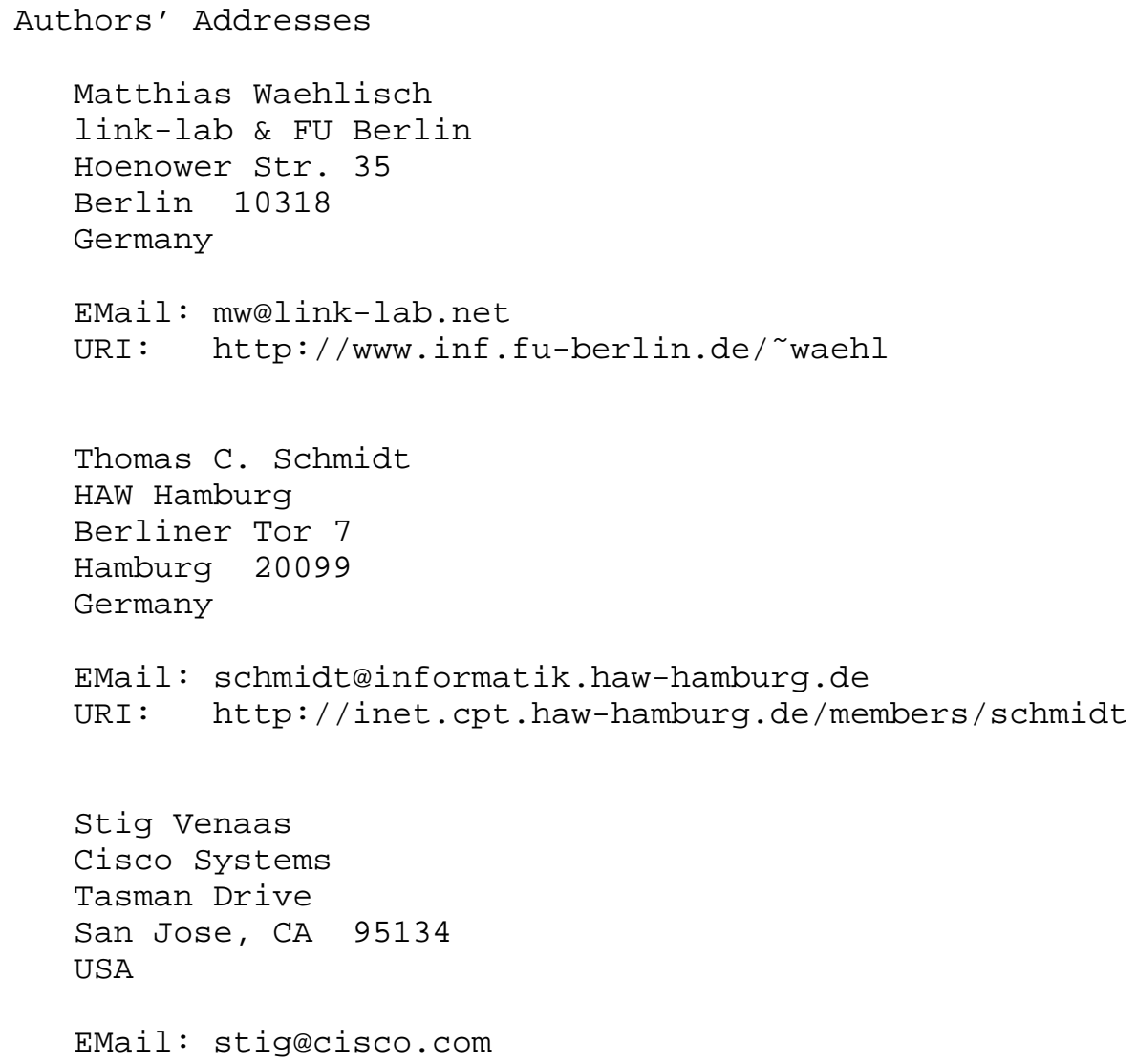

